Diagnostics and Physics Contributions by The University of Texas Fusion Research Center to the HT-7 and HT-7U Tokamak Programs of the Institute of Plasma Physics, Chinese Academy of Sciences

DOE Award Number DE-FG02-03ER54729

\title{
Closeout Technical Report
}

Dr. Kenneth W. Gentle

Principal Investigator

November 29, 2004

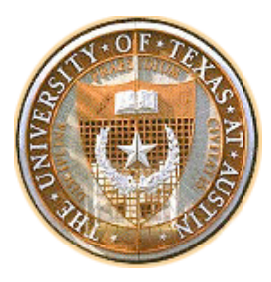

Fusion Research Center

THE UNIVERSITY OF TEXAS AT AUSTIN

1 University Station C1500, Austin, TX 78712-0262 • 512-471-4318 


\subsection{INTRODUCTION}

We are conducing a collaborative research program on two tokamaks, HT-7 and EAST (Experimental Advanced Superconducting Tokamak, formerly HT-7U), with the Institute of Plasma Physics of the Chinese Academy of Sciences (CASIPP) located in Hefei, PRC. The work that we planned for this year included conducting transport experiments on HT-7, completing plans for expansion of the HT-7 diagnostic set and reaching an agreement on how UT-FRC can best participate in experiments on HT-7U. These goals were accomplished as summarized in the next section. Note that the experimental portion of the work is still underway. The experimental campaign for HT-7 began just a few weeks before this report was compiled.

\subsection{ACCOMPLISHMENTS}

\section{ECE RADIOMETER}

An additional eight channels were added to the UT-FRC ECE radiometer installed on HT-7 to extend measurements of $T_{e}$ from the high field side of the plasma to the low field side of the plasma. This facilitates thermal transport experiments and allows investigation of suspected auxiliary heating asymmetries.

The signal-to-noise ratio and the stability of the calibrations were improved by locating the electronics well away from the tokamak in a temperature-controlled rack. The use of a temperature controlled was successful on our ECE radiometer at DIII-D and will be applied at C-Mo prior to the next campaign.

The HT-7 run campaign began in late April, only a few weeks before this report was compiled. The entire 16-channel system is operating (see the detailed report). We are awaiting discharges suitable for our research goals.

An ASIPP staff member was assigned to be responsible for day-to-day operation of the radiometer. Staff at UT-FRC in Austin maintain the system calibration and assist with data analysis via remote access. A UT-FRC engineer is on-site during campaigns to assure proper operation of the diagnostics. 


\section{DNB TRANSFER to ASIPP}

Formed a DNB team which will continue work following this grant closeout provided there is continued support from DOE. The team consists of

Wan, Shaohu Project Organiser, IPP side

Huang, He Project Organiser, FRC side

Liu, Baohua $\quad$ Power Team Leader

$\mathrm{Hu}$, Chengdong Beam Line Team Leader

$\mathrm{Hu}$, Liquen $\quad$ Physics Team Leader

Power Team: 4 IPP Engineers, 1 Contract Engineer, 3 Technicians. 1 FRC Engineer

Beam Line Team: 3 IPP Vacuum Engineers, 3 IPP Electronic Engineers, 2 Scientists.

Physics Team: 1 IPP Scientist, 1 FRC Scientist.

A DNB formerly used on TEXT has been transferred to ASIPP for use on HT-7. The DNB will be effective for the low density plasmas generated in that device.

The DNB was shipped to Hefei. The last shipment arrived in October 2003.

UT-FRC participated in the design of the HT-7 installation and transferred documentation to ASIPP.

A UT-FRC staff member is coordinating the beam installation. ASIPP has assigned an engineering team with members responsible for beam line assembly, DNB control, and DNB power supplies.

A UT-FRC staff member collaborated with an ASIPP staff member on the design of the spectroscopic monitor for the beam.

Finished the high voltage power supply modification, installed deionized water system and created 60KV pulse in September 2004. 
Completed power system integration test with all power supplies operating into dummy loads in November 2004.

Evaluation of DNB transmission inside HT-7 plasma in cooperation with FRC scientist was essentially completed in May 2004.

Design and manufacture of beam transport duct and port for beam specides mix was finished in October 2004.

Design and execution of optics for beam component mix. Design completed in October 2004. Evaluation of design is underway with the expectation that it will be completed by the end of the year under condinued DOE funding.

Design and manufacture of carbon armor tile for DNB beam shine-through. Design was finished in October 2004. Manufacturer started.

Optical design for poroidal and toroidal CXRS systems, conceptual design finished in May 2004. Detailed design and choice of optical components is by an FRC scientist and will be completed in December 2004 under continued DOE funding.

\section{CXRS}

The conceptual design for a 20 channel CXRS system was completed. The diagnostic will have both poloidal and toroidal channels. The detailed design is underway.

An ASIPP staff member is assigned to collaborate on the design of the CXRS system.

\section{MOTIVATED THE IMPLEMENTATION OF MDSPLUS AT HT-7}

A UT-FRC staff member provided local tutorials on MDSPlus and then facilitated communication with US experts. This resulted in the archiving of HT-7 data in MDSPlus on a trial basis.

This is an advantage for UT-FRC as we have better access to data and can use our standard computational methods for analyzing data. We expect this example to be noted by our ASIPP colleagues and that they will then adopt MDSPlus for both HT-7 and EAST. 


\section{Agreements on Future Work}

A major goal for this year's work is to formalize out interactions with ASIPP to the extent of having specific areas of contribution to the HT-7 and EAST programs. The DNB, CXRS, and radiometer work mentioned above are the bulk of the results of these agreements for HT-7.

UT-FRC has been invited to participate in the development of a Doppler reflectometer. The purpose of the device would be the measurement of phase rotation of broadband fluctuations. We have made some contributions to the conceptual design of this device, but cannot pursue it at our present funding level. This issue is discussed in the proposal for our next fiscal year.

UT-FRC will transfer the ECE radiometer and the CXRS system to EAST with the necessary upgrades to make the diagnostic effective on that device. This will be the subject of the next proposal period.

The DNB would require significant upgrades for the transfer to EAST. UT-FRC will provide the engineering coordination for this transfer including grid refocusing, addition of a magnetic filter to the source to improve component mix, redesign of power supply packaging to reduce size of the DNB. These major upgrades and may require replacement of some subsystems. This will be the subject of the next proposal period.

\section{Travel and Attendance at Meetings}

Four staff members of UT-FRC traveled to Hefei, PRC in October 2003 to work on the ECE radiometer, to begin the DNB project, to participate in the 4th General Scientific Assembly of Asia Plasma \& Fusion Association (APFA) Conference, Hangzhou, PRC, and to participate in the EAST International Advisory Committee Meeting, Hefei, PRC.

Three staff members of UT-FRC traveled to Hefei, PRC to prepare diagnostics for the Spring HT-7 experimental campaign and to consult on the DNB installation. The beam vacuum system was brought into operation. A provisional design for the CXRS system was completed. 


\section{Publications and Presentations}

"Recent Progress on the steady-state- high performance plasma research in HT-7," Baonian Wan, BAPS 48, 83 (2003). Invited talk at the APS-DPP annual meeting with UT-FRC acknowledged as a contributor.

"Neutral Beam Diagnostics for the HT-7 Tokamak," L.Q. Hu, B.N. Wan, C.D. Hu, B.H. Liu, Z.W. Wu, J. Huang and HT-7 DNB Team, William L. Rowan, H. He, K. Gentle, to be published in Rev. Sci. Instrum.

"The Experiment of Modulated Toroidal current on HT-7 and HT-6M Tokamak," Jian-shan Mao, P. Phillips, .et. al., Plasma Science \& Technology, 5, No. 1, 2003

"Direct Measurements of the Electron Energy Flux versus Electron Temperature Gradient in Tokamak Discharges", K.W. Gentle, M.E. Austin, P.E. Phillips, 4th General Scientific Assembly of Asia Plasma \& Fusion Association (APFA) Conference, Hangzhou, China, October 13-16, 2003

\subsection{ECE RADIOMETER FOR HT-7 AND EAST}

During the last experimental campaign on HT-7, we installed an ECE radiometer and brought it into operation as a standard diagnostic and as a tool for our own research. Since then, we have upgraded the system by doubling the number of channels to a total of sixteen. This was done with the close cooperation of ASIPP, and they have committed a postdoc to operate the system. The system can be transferred to EAST when that device begins operation. We will continue to improve the existing system to provide the best possible radiometer for EAST.

The current campaign on the smaller device, HT-7, which began in late April is our first opportunity to do experiments with the sixteen channel system. During this campaign, we intend to exploit the system for collaborative research in three areas 
- heat pulse transport using sawtooth heat pulse propagation

- heat pulse transport using IBW as the pulsed heat source

- mode destabilization due to Lower Hybrid Current Drive and subsequent stabilization with Ion Bernstein Wave Heating.

In figure 1, the profile coverage is given for various HT-7 magnetic fields. The vertical axis shows the toroidal magnetic field at the axis which is $1.22 \mathrm{~m}$ in HT-7. This narrow range of magnetic field covers the range of variation employed for this device. On the horizontal axis, the inner and outer major radius (the limits of the plasma due to the vacuum vessel) are shown. The shaded area of the left shows the radial coverage of the system that we now have installed. Note that for the nominal $2 T$ field, the FRC ECE system covers the high field side of the entire plasma.

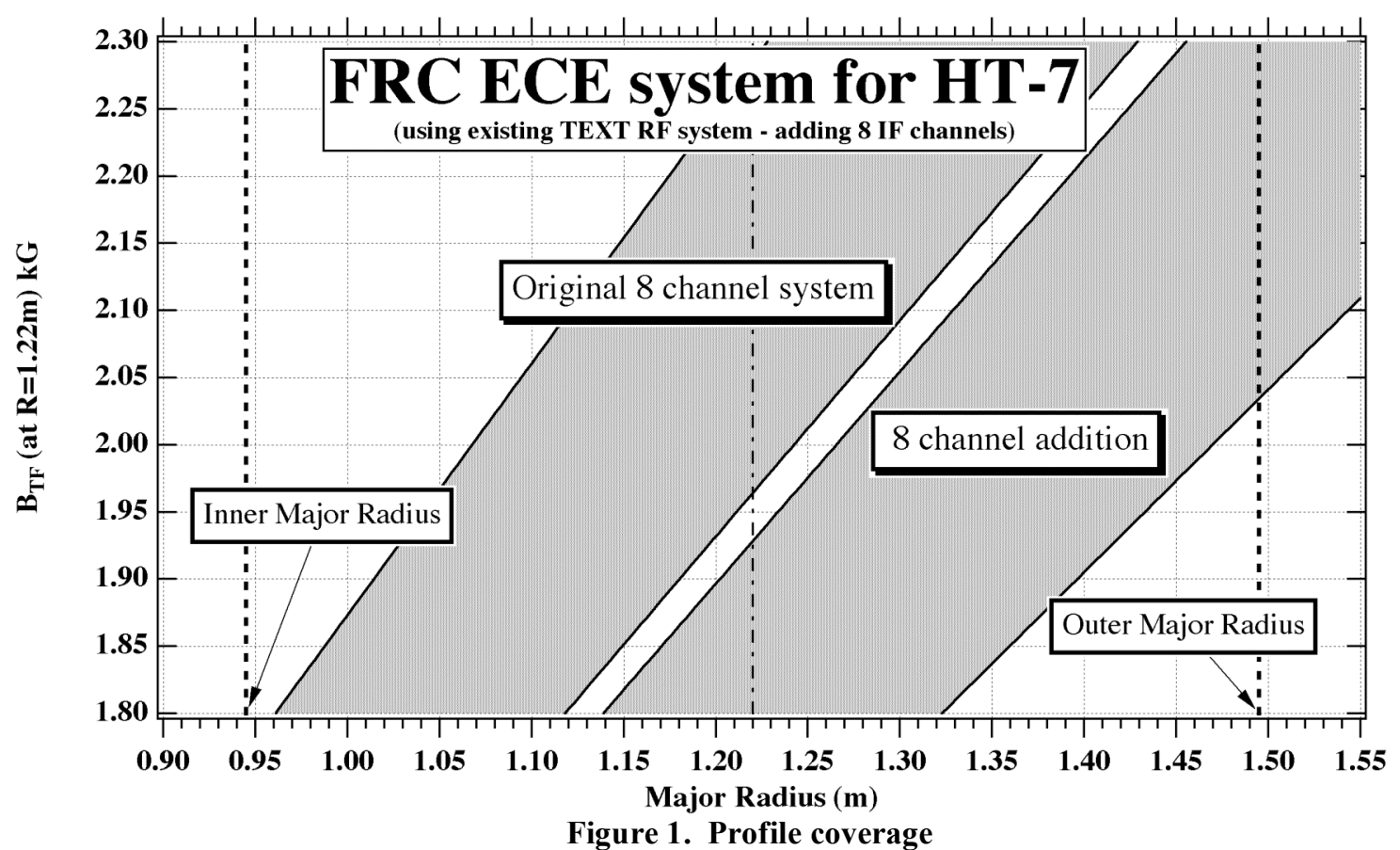

An example of the data acquired with this system at this early stage of the current campaign is shown in the following two figures. In the first of these, Figure 2, the time series for each channel is shown. Taken during the second week of plasma operation, this data still shows some excess noise and the signal levels are somewhat small. A new video amp has recently been installed and will likely improve that. 


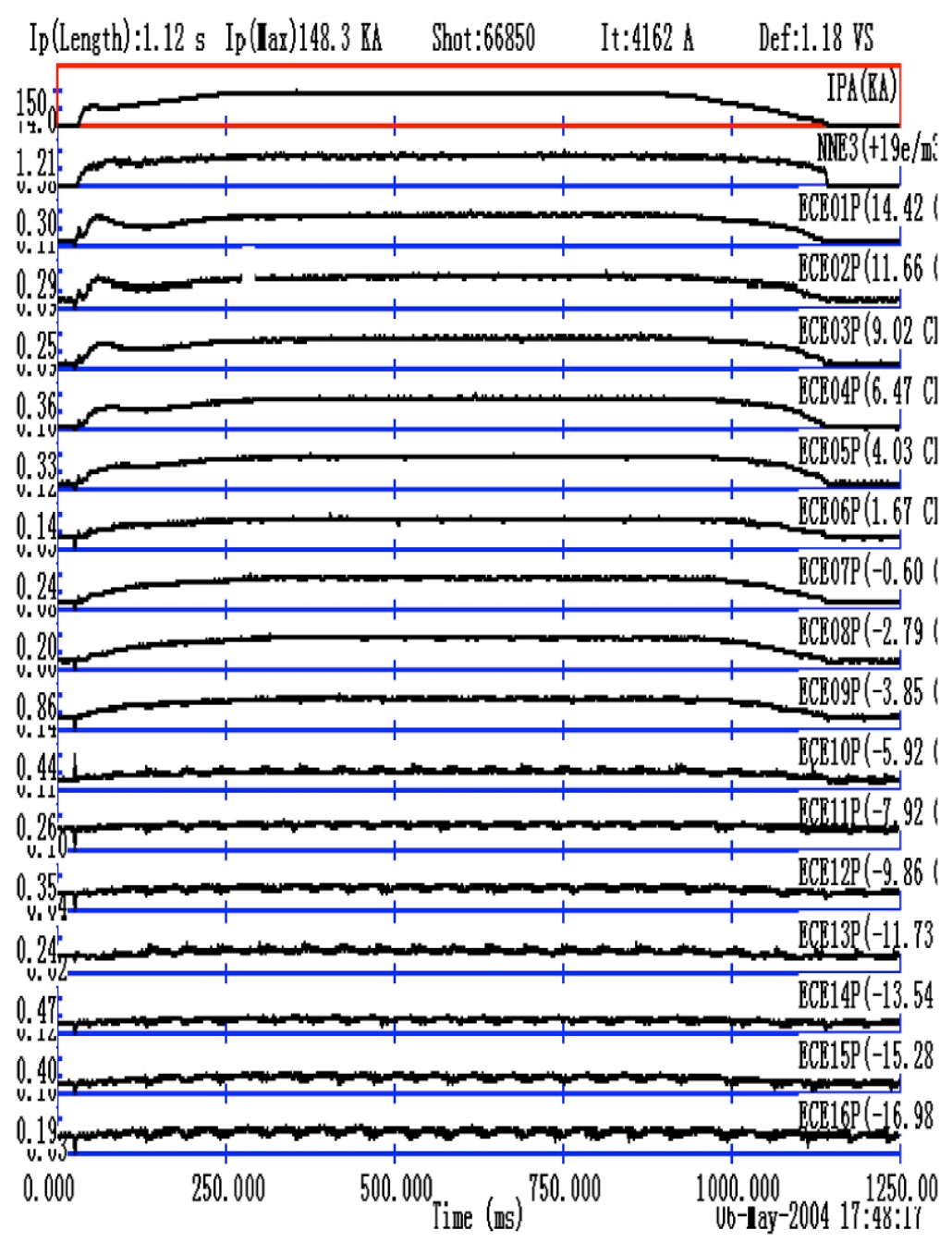

Figure 2. Time series data from the sixteen-channel ECE radiometer

The data can be used to develop profiles. One of these is shown in Figure 3. 


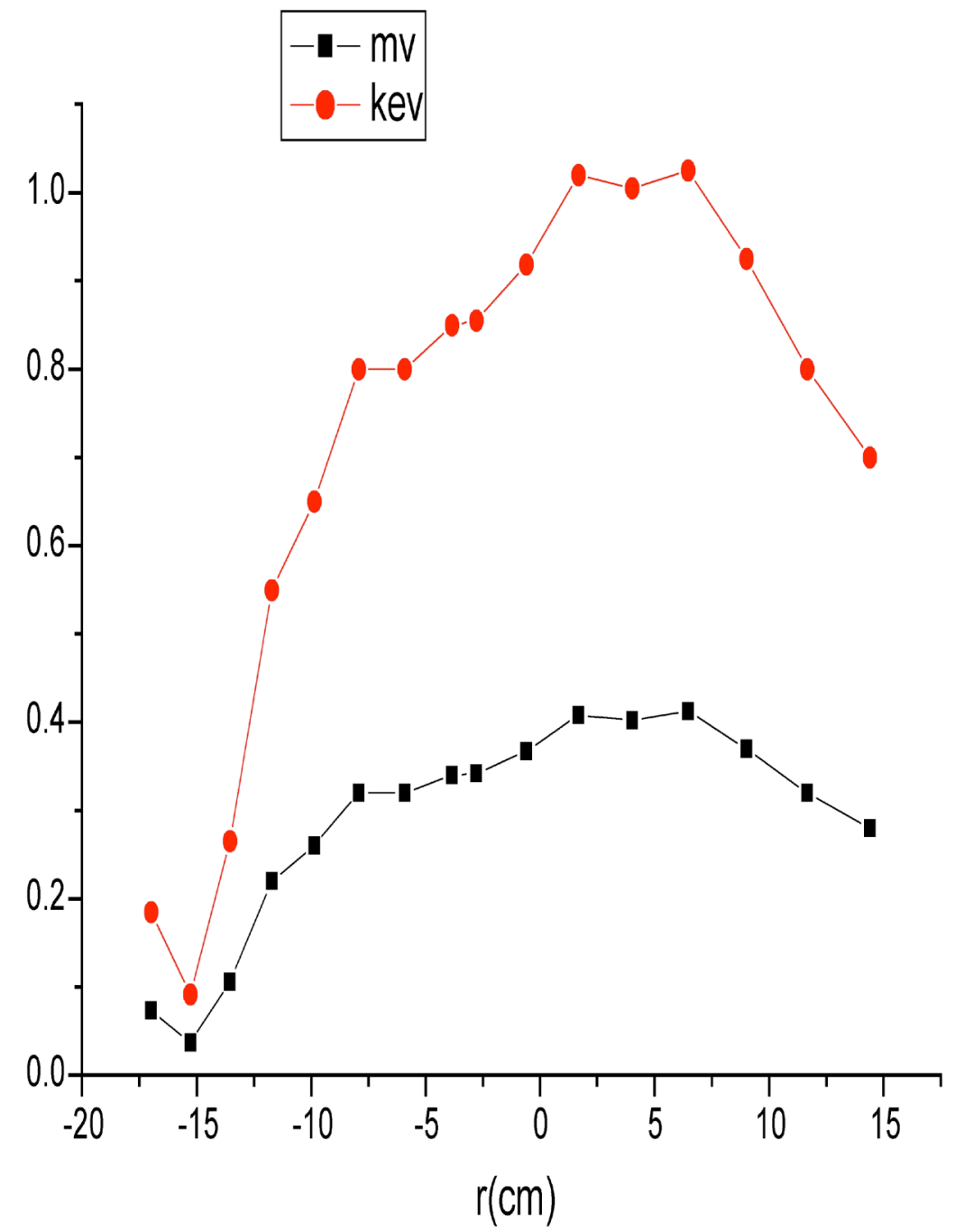

Figure 3. Te profile for HT-7. The red points represent the signal in $\mathrm{mV}$. The black points represent temperature with a preliminary calibration.

The profile still demonstrates beginning-of-campaign malaise. It is quite clear though that profiles will be available shortly and that we will be able to start the pursuit of our research goals.

\subsection{CXRS, DNB Installation, ANd Spectroscopic DNB Monitor FOR HT-7}

A DNB was transferred to ASIPP at the end of 2003. The beamline is now assembled and we expect to conduct tests on the plasma source in mid June. This will be followed by 
installation on HT-7 for the fall campaign. A CXRS system will be installed at the same time. UT-FRC is providing engineering and physics support for the beam installation and for prediction of its performance. We plan to design the CXRS system, assemble it at UT-FRC and then transfer it to HT-7. Also, we are designing and constructing a beam monitor for automatic measurement of the beam component mix on each shot. The conceptual design for the beam installation, for the CXRS system, and for the spectroscopic beam monitor are described in this section.

\section{DNB INSTALLATION}

The beam that was transferred was built at Culham labs and was the used on TEXT and in early DNB work on Alcator C-Mod. This beam is well known to be useful in low density plasmas such as that on HT-7. (see Figure 4).

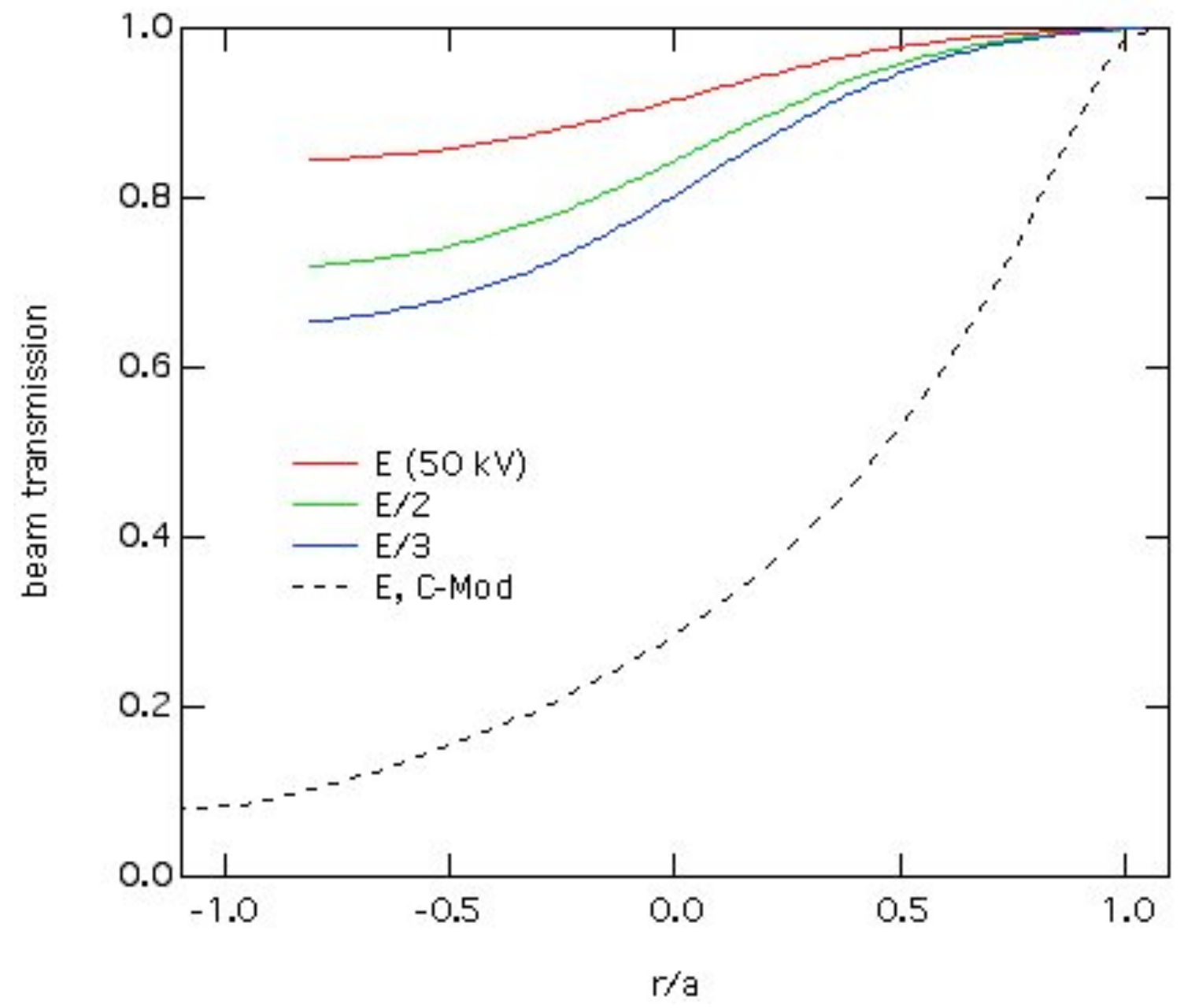


Figure 4. Beam transmission predicted for HT-7 and a comparison to its operation on C-Mod. While the beam operation was problematic on C-Mod, it should be quite acceptable on HT-7.

In normal operation, the DNB can produce $5.5 \mathrm{~A}$ of extracted beam current in hydrogen at an energy of $50 \mathrm{keV}$ with a pulse length of $0.1 \mathrm{~s}$. The measured density fractions in the neutralized beam are typically $[\mathrm{E}: \mathrm{E} / 2: \mathrm{E} / 3: \mathrm{E} / 18]=[0.12: 0.34: 0.37: 0.17] . \mathrm{D}$ and He beams can be produced. The beam can be modulated at $0.5 \mathrm{kHz}$. The installation that we plan is displayed in Figure 5.

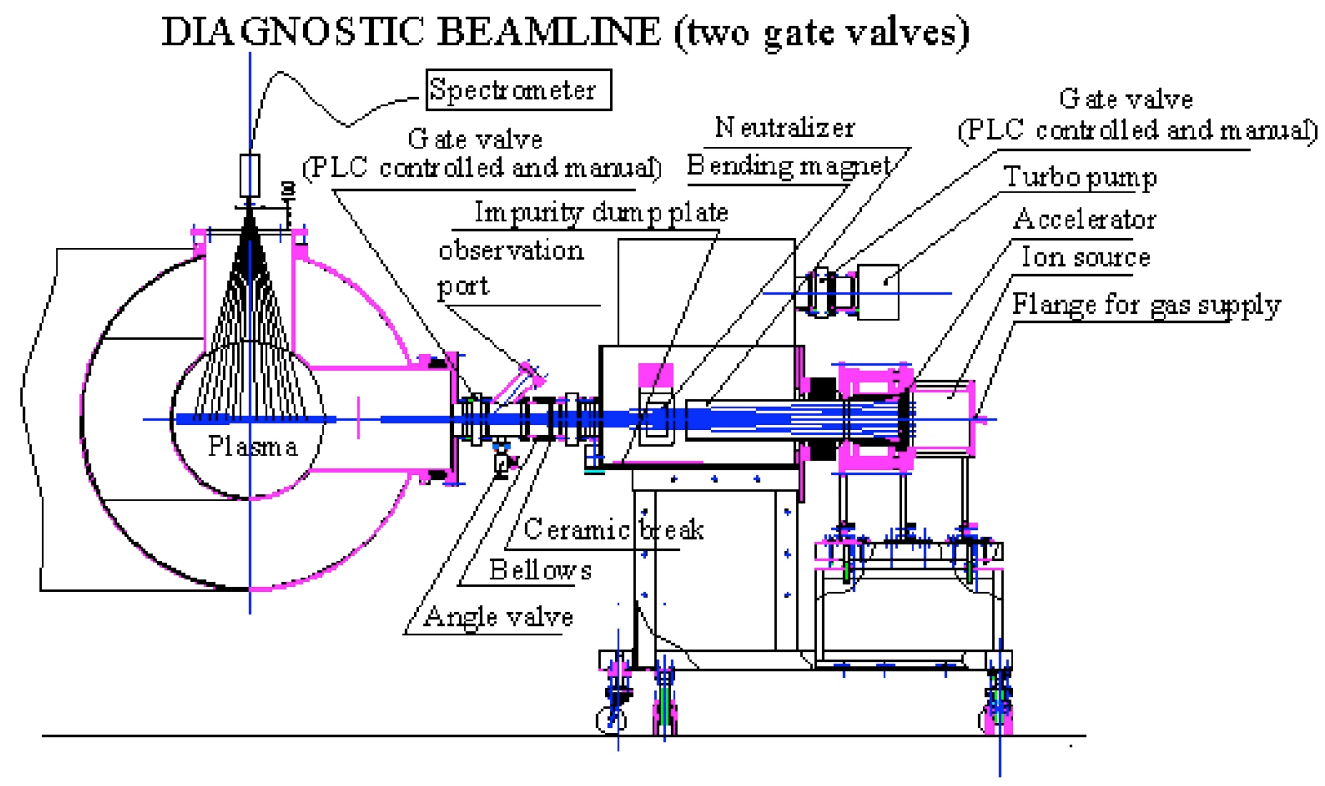

Figure 5. Installation of the DNB on HT-7. Details of the beam duct are shown. Also, the poloidal chords for the CXRS system are shown.

At present, the beam is being assembled and tested in a lab near the HT-7 facility (see Figures 6 and 7. 


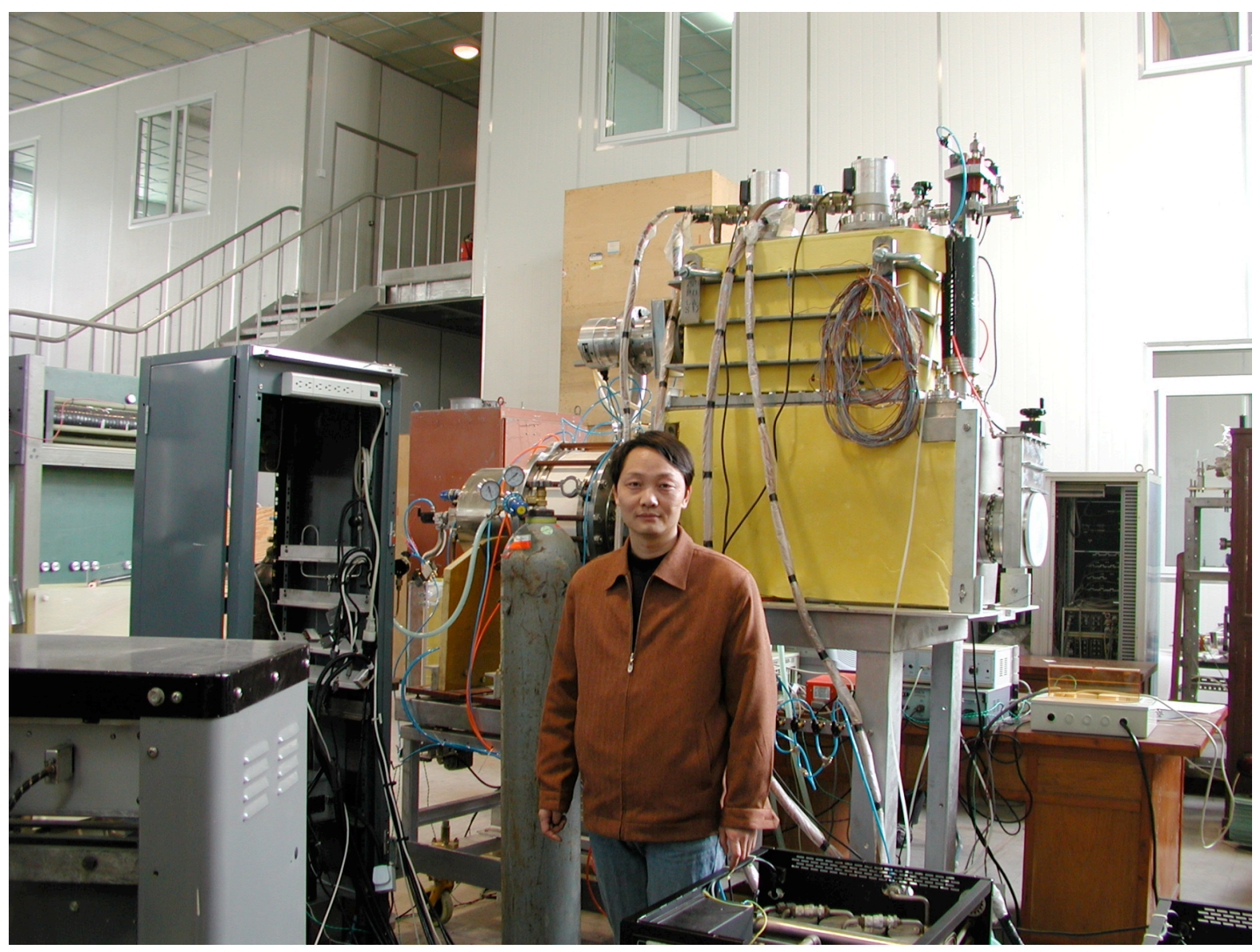

Figure 6. The assembled beam line is shown in the background. In the foreground is C. D. Hu, the lead CASIPP engineer for the beam. February 2004 


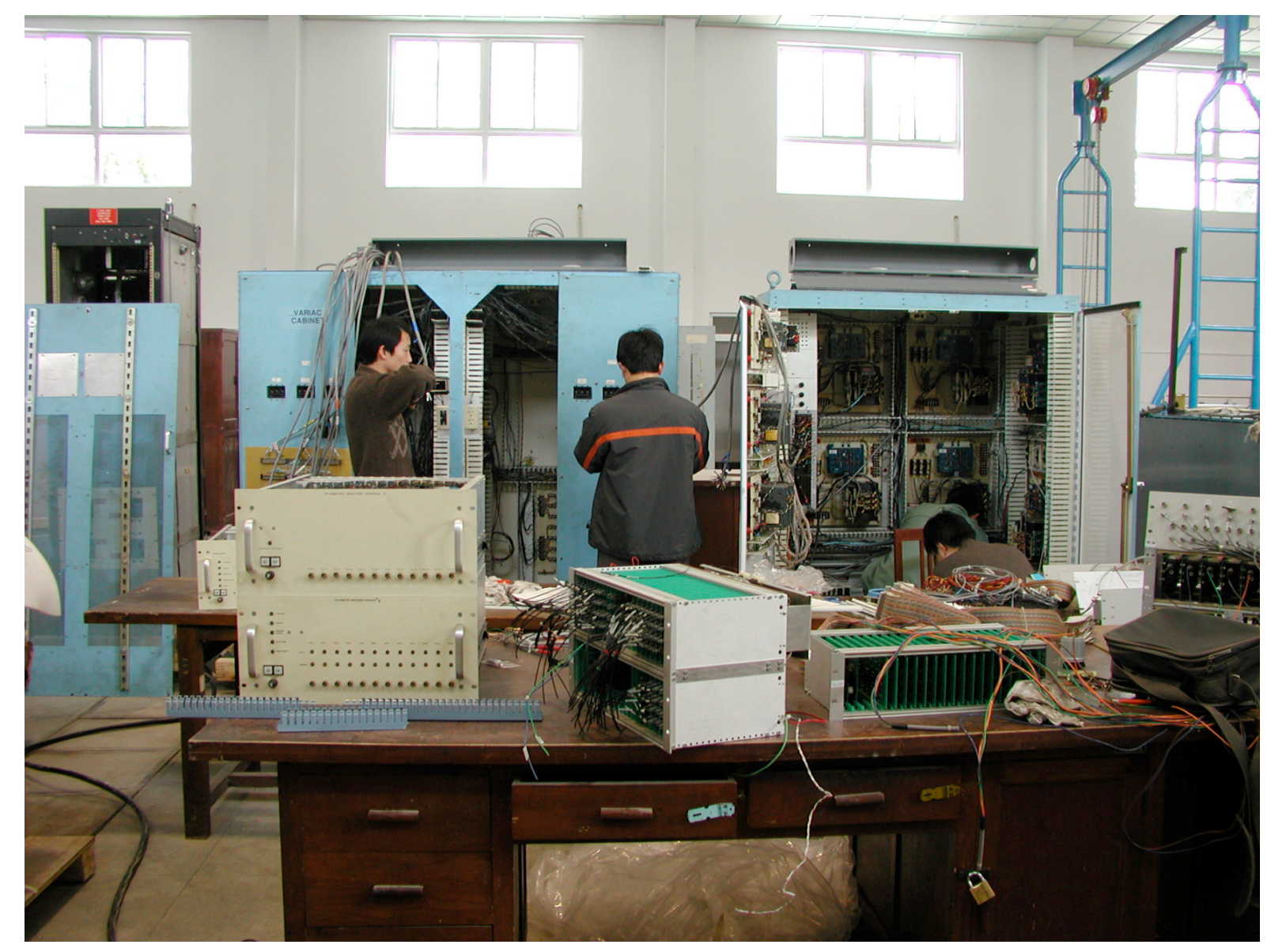

Figure 7. CASIPP engineers modifying the beam primary power cabinets for integration into the HT-7 power systems.

\section{CXRS}

Since the limiter material is silicon-doped carbon and boronization is the routine wall conditioning method in the HT-7 tokamak, CXRS measurements will be attempted with both carbon $\left(C^{+5}, n=8 \rightarrow 7, \lambda \approx 529.1 \mathrm{~nm}\right)$ and boron $\left(B^{+4}, n \approx 7 \rightarrow 6, \lambda \approx 494.6 \mathrm{~nm}\right)$.

One toroidal and one poloidal optical CXRS system will be implemented at the outset. The poloidal system is shown in Figure 5. The toroidal system will be one of those appearing in Figure 8. The two options are left open because we are not to the point in the implementation to know what space is available and whether the required optical etendue is available form both locations. The target resolution is $2 \mathrm{~cm}$. The design of the spectrometer detector system will be based on the existing forty-five channel system designed by UT-FRC and installed on Alcator CMod. It is likely that changes will be required to improve spatial resolution. 


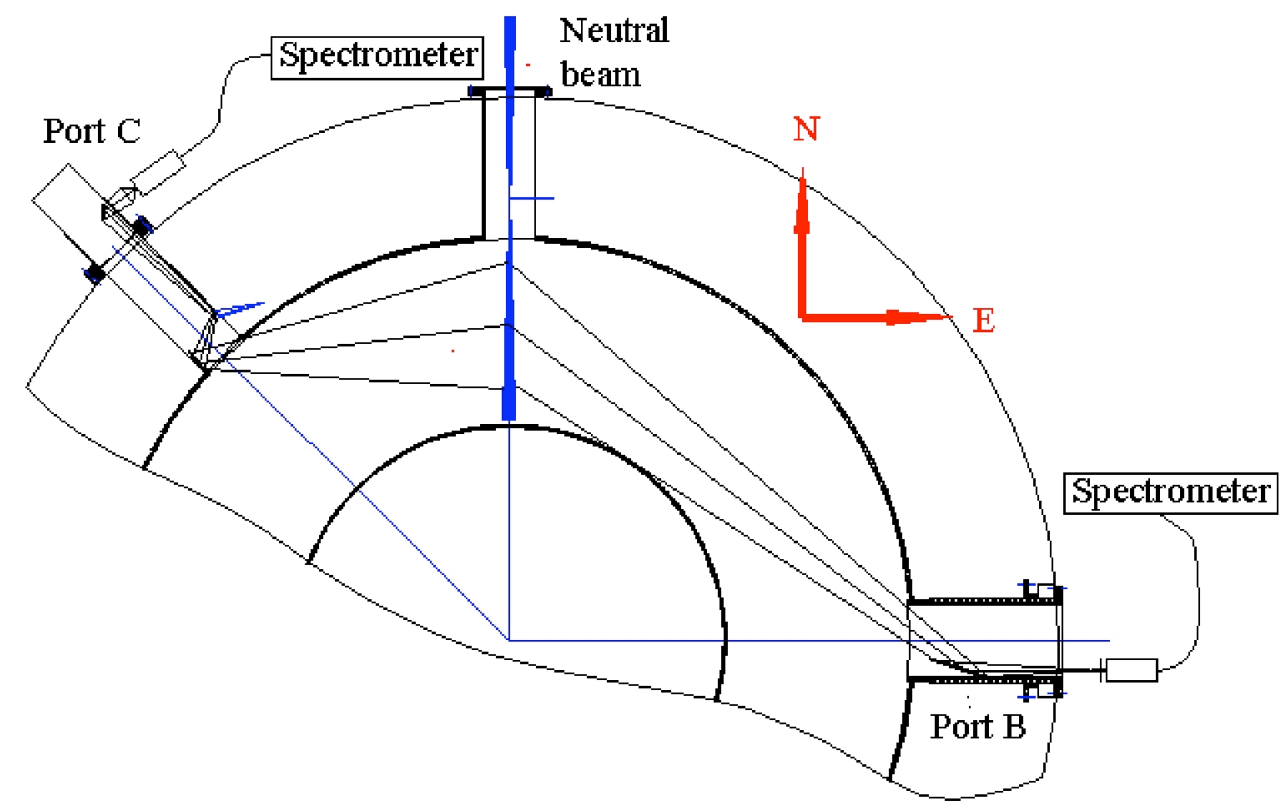

Figure 8. The CXRS spectrometer will be located at one of two ports depending on the results of the design study.

\section{A Spectroscopic Beam Monitor}

For a DNB, it is useful to have a measure of the beam component mix and beam parameters on essentially every shot for tuning the beam and to contribute to understanding of the diagnostic signals. UT-FRC will design and implement a beam monitor for the DNB installed on HT-7.

The beam emission will be observed as indicated in the schematic of Figure 9.

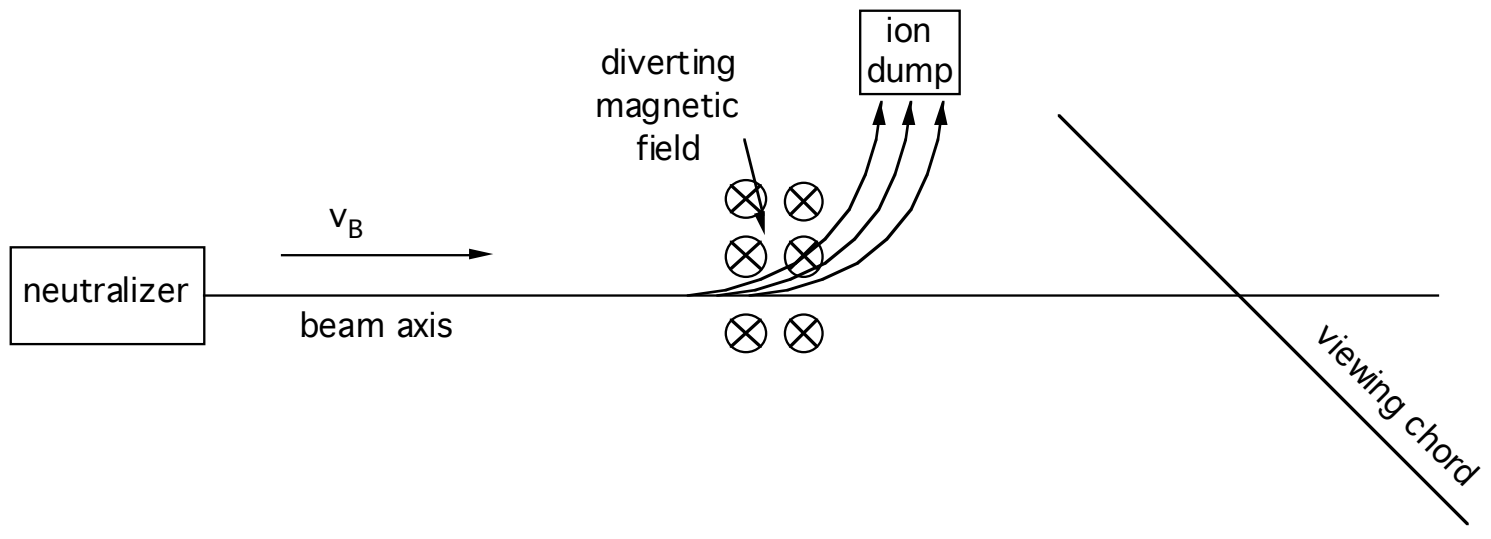

Figure 9. Schematic of the spectroscopic beam component measurement 
A spectrometer will view the beam along a chord that such as the viewing chord in Figure 9. The beam will be viewed downstream from the neutralizer and from the diverting magnet so that the beam is neutral and contains an insignificant density of ions. The expected spectrum will be similar to that of Figure 10 (which was taken by UT-FRC staff for a beam operating on Alcator C-Mod.

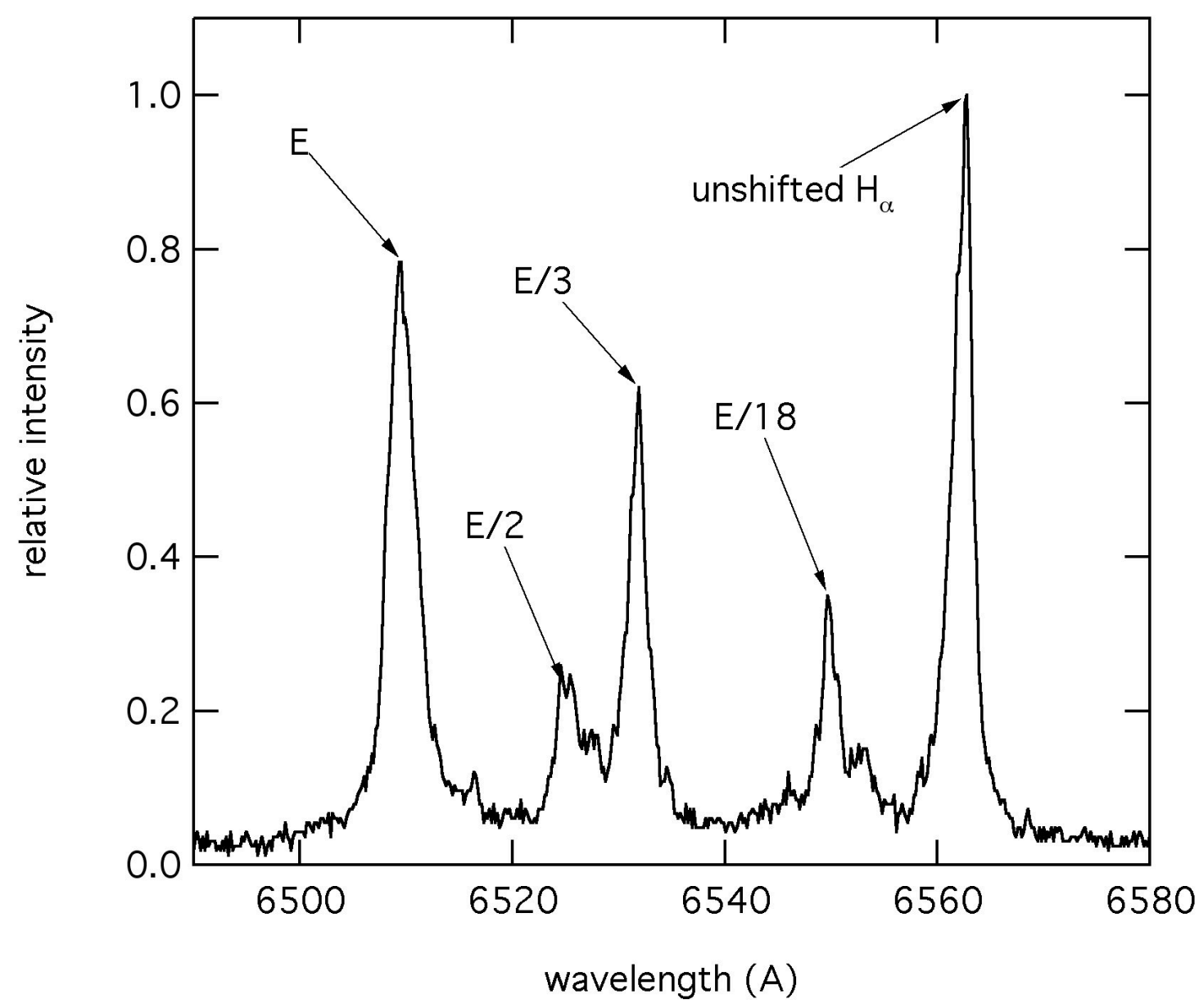

Figure 10. A measured beam spectrum. The unshifted $H_{\alpha}$ is emitted by the background $H_{2}$. A Doppler shifted $\mathrm{H}_{\alpha}$ is emitted by each of the beam energy components. When interpreted as described in the text this spectrum yields a beam density mix of $(E: E / 2: E / 3: E / 18)=(0.45: 0.08: 0.29: 0.18)$.

The spectrum that will be acquired for the DNB on HT-7 will have a lower resolution but should be just as effective in obtaining results that are useful for interpreting beam component mix. A simulation of the expected spectrum is in Figure 11. The spectrometer intended for this work is an Acton Research Spectral Pro 300i. The density component mix is taken to be $(\mathrm{E}: \mathrm{E} / 2: \mathrm{E} / 3: \mathrm{E} / 18)=(0.22,0.30,0.43,0.04)$ which is typical for the beam in use. 
The cross sections for emission are taken from the measurements found in Williams, Geddes, and Gilbody, J. Phys. B 15, 1377(1982). The emission is aperture broadened (L. C. Marquet, JOSA 57, 878 (1967). This analysis indicates that the diagnostic will work. During the next proposal period, it will be implemented and brought into operation.

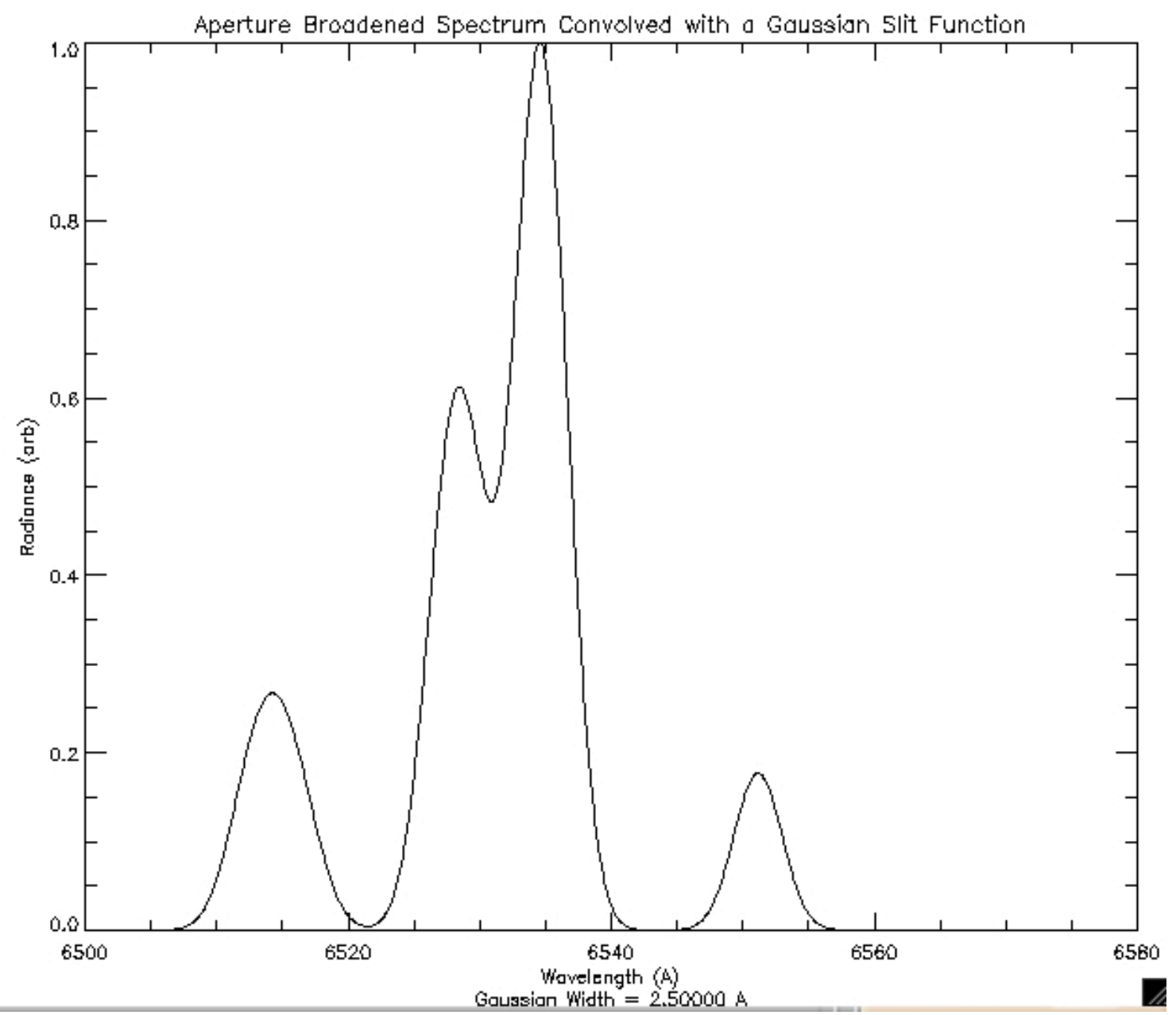

Figure 11. The simulated spectrum produced by the beam component mix diagnostic. 